\title{
gravity on Riemann surfaces in conformal gauge
}

ARTICLE in CLASSICAL AND QUANTUM GRAVITY · JULY 1997

Impact Factor: 3.17 · DOI: 10.1088/0264-9381/14/7/015

\section{AUTHOR:}

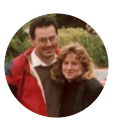

Paolo Valtancoli

University of Florence

52 PUBLICATIONS 408 CITATIONS

SEE PROFILE 
$(2+1)$ gravity on Riemann surfaces in conformal gauge

This content has been downloaded from IOPscience. Please scroll down to see the full text. 1997 Class. Quantum Grav. 141795

(http://iopscience.iop.org/0264-9381/14/7/015)

View the table of contents for this issue, or go to the journal homepage for more

Download details:

IP Address: 194.190.160.201

This content was downloaded on 13/03/2016 at 17:39

Please note that terms and conditions apply. 


\title{
$(2+1)$ gravity on Riemann surfaces in conformal gauge
}

\author{
P Valtancoli \\ Dipartimento di Fisica dell' Universita', Firenze and INFN, Sezione di Firenze, Italy
}

Received 27 November 1996, in final form 13 February 1997

\begin{abstract}
We derive a first-order formalism for solving $(2+1)$ gravity on Riemann surfaces, analogous to the recently discovered classical solutions for $N$ moving particles. We choose the York time gauge and the conformal gauge for the spatial metric. We show that Moncrief's equations of motion can be generally solved by the solution $f$ of a $O(2,1) \sigma$-model. We build out of $f$ a mapping from a regular coordinate system to a Minkowskian multivalued coordinate system. The polydromy is in correspondence with the branch cuts on the complex plane representing the Riemann surface. The Poincaré holonomies, which define the coupling of Riemann surfaces to gravity, simply describe the Minkowskian free motion of the branch points. By solving $f$ we can find the dynamics of the branch points in the physical coordinate system. We check this formalism in some cases, i.e. for the torus and for every Riemann surface with $S O(2,1)$ holonomies.
\end{abstract}

PACS numbers: 0460K, 1125H, 0420, 0420G, 0420J, 0350

\section{Introduction}

In this paper we will study three-dimensional gravity as an empty universe. Since there are no gravitons the only dynamical degrees of freedom of pure $(2+1)$ gravity are topological, i.e. Riemann surfaces evolving in time. The reason for such an investigation is essentially to understand the peculiar features of quantum gravity in an integrable system $[1,6]$.

At the classical level there has been a lot of work in the Hamiltonian formalism, selecting the appropriate foliation on which the solution can exist without spurious singularities [6]. Some particular solutions have been found, for example in the torus case, whose modulus, $\tau$, describes a circular motion in the Teichmuller space $[6,8]$.

At the quantum level since $(2+1)$ gravity has a finite number of physical degrees of freedom quantum field theory can be effectively reduced to quantum mechanics. For example we can quantize the modulus Lagrangian of a torus, introducing a canonical momentum to $\tau$, and writing down a Schrödinger equation acting on the Hilbert space of square integrable functions of $\tau$ [8]. This reduced quantization is analogous to the particle case [4], where one can integrate out the field into an effective action for the particle degrees of freedom. However, there is no clear picture of how to deal with secondquantized processes, i.e. for example how to treat topology-changing amplitudes or creation and annihilation processes for particles [1].

Maybe a new treatment of three-dimensional gravity resembling two-dimensional quantum field theory can handle these problems. At the classical level particle dynamics has already been solved in the gauge $K=0$ [9] only because this gauge makes possible extensive use of the complex variable and the vast knowledge on conformal mappings. Exact results have been found in the two-body case [10], and an interesting connection 
with Painlevé VI for the three-body case [10-12]. Furthermore, Ciafaloni has proposed extending this gauge to higher topologies and he found an explicit solution for the torus case [13].

In this paper we investigate how to generalize our first-order formalism, which has been by far the simplest method to deal with particle scattering, in order to treat topological degrees of freedom. Moncrief has shown that a good time slicing for topology is the York time slicing ( $K$ constant but not zero). The resulting equations of motions can be simplified by choosing a conformal gauge for the spatial metric. Each spatial slice is equivalent to a Riemann surface, which we choose to represent with branch cuts on the complex plane. The corresponding metric is singular at the branch points, which move as particle singularities.

The property of instantaneous propagation of the fields still holds in this gauge, and the field dynamics can be reduced to a two-dimensional field theory. It turns out that instead of having a Liouville field theory, which is classically solved by a conformal map $f(z)$, we have to deal with a sinh-Gordon model, which can be solved by a solution, $f(z, \bar{z})$, of the $O(2,1) \sigma$-model.

We will analyse the general properties of this map $f$, giving some explicit solutions in several cases. We will build out of $f$ a multivalued mapping $X^{a}=X^{a}(x)$ from a regular coordinate system to a multivalued Minkowskian coordinate system. The motion of the branch points in the $X^{a}$ coordinates is free and determined by the Poincare holonomies, defining the coupling of the Riemann surface to gravity. By solving the mapping $f$, we can find the dynamics of the branch points and of the moduli of the Riemann surface in the physical coordinate system.

In section 2 we give as reference the York time gauge in the second-order formalism. In section 3 we introduce the first-order formalism and we explain its connection with the $O(2,1) \sigma$-model. In section 4 we give some explicit solutions with which we can check this formalism and explain the qualitative behaviour of the general solution. In section 5 after reviewing our results we give some concluding remarks. In the appendix we give the proof how to derive from the $O(2,1) \sigma$-model Moncrief's equations of motion.

\section{York time gauge in the second-order formalism}

We shall work in the $A D M$ formalism, assuming that spacetime can be globally decomposed as $\Sigma(t) \otimes R$, where $\Sigma(t)$ is a set of spacelike surfaces [13,14]. We take as parametrization for the metric:

$$
\mathrm{d} s^{2}=\alpha^{2} \mathrm{~d} t^{2}-\mathrm{e}^{2 \phi}|\mathrm{d} z-\beta \mathrm{d} t|^{2},
$$

where we have chosen conformal coordinates for the spatial metric. This choice of variable, the lapse function $\alpha$ and the shift functions $\beta$, is particularly useful when we discuss how to solve the Eulero-Lagrange equations of motion.

Let us recall the $A D M$ decomposition of the Einstein-Hilbert action into a spatial part, intrinsic to the surfaces $\Sigma(t)$, and an extrinsic part, coming from the embedding, as follows

$$
S=-\frac{1}{2} \int \sqrt{|g|} R^{(3)} \mathrm{d}^{3} x=-\frac{1}{2} \int \sqrt{|g|}\left[R^{(2)}+(\operatorname{Tr} K)^{2}-\operatorname{Tr}\left(K^{2}\right)\right] \mathrm{d}^{3} x,
$$

The extrinsic curvature tensor, $K_{i j}$, or second fundamental form of the surface $\Sigma(t)$, is given in terms of the covariant derivatives $\nabla_{i}^{(2)}$ with respect to the spatial part of the metric:

$$
K_{i j}=\frac{1}{2} \sqrt{\frac{\left|g_{i j}\right|}{|g|}}\left(\nabla_{i}^{(2)} g_{0 j}+\nabla_{j}^{(2)} g_{0 i}-\partial_{0} g_{i j}\right) .
$$


Our aim is to simplify the Moncrief program for solving $(2+1)$ gravity on Riemann surfaces. The choice of conformal coordinates for the spatial metric allows us to represent a Riemann surface on a complex plane with branch points (four branch points for a torus, $2 g+2$ for an hyperelliptic surface of genus $g$, and so on). This means that the metric has a singular particle-like behaviour on such branch points, which, however, must satisfy some integrability condition, such as a finite area $A(t)=\int \mathrm{d} z \mathrm{~d} \bar{z} \mathrm{e}^{2 \phi}$ on each spatial slice.

The Lagrangian for $(2+1)$ gravity, restricted to a spatial metric in conformal gauge, is defined by:

$$
\begin{aligned}
& \mathcal{L}=\alpha \nabla^{2} \phi+\alpha \mathrm{e}^{2 \phi} K^{2}-\frac{\mathrm{e}^{2 \phi}}{\alpha}\left|\partial_{z} \bar{\beta}\right|^{2} \\
& K=-\frac{1}{2} g^{i j} K_{i j}=\frac{\mathrm{e}^{-2 \phi}}{2 \alpha}\left[\partial_{z}\left(\beta \mathrm{e}^{2 \phi}\right)+\partial_{\bar{z}}\left(\bar{\beta} \mathrm{e}^{2 \phi}\right)+\partial_{0} \mathrm{e}^{2 \phi}\right] .
\end{aligned}
$$

A simplifying feature appears in the equation of motion for $\beta$, which reads

$$
\partial_{\bar{z}} N+\mathrm{e}^{2 \phi} \partial_{z} K=0, \quad N=\frac{\mathrm{e}^{2 \phi}}{2 \alpha} \partial_{z} \bar{\beta} .
$$

From this equation it is clear that is $N$ is conformal whenever $K=0$ or $K$ is a timedependent constant. In general, we can always choose to define the time coordinate such that

$$
K=(2 t)^{-1} \rightarrow \partial_{\bar{z}} N=0 .
$$

Therefore, our gauge choice is defined by the conditions

$$
g_{z z}=g_{\overline{z z}}=0 \quad K=(2 t)^{-1},
$$

and thus corresponds to a conformal gauge, with York time $g_{i j} K^{i j}=-(t)^{-1}$.

The above conditions are enough to eliminate time derivatives from the Lagrangian and to give an instantaneous propagation of the metric, as it appears from the Eulero-Lagrange equations for $\alpha$ and $\phi$ :

$$
\begin{aligned}
& \nabla^{2} \phi-\frac{1}{4} t^{-2} \mathrm{e}^{2 \phi}+4 N \bar{N} \mathrm{e}^{-2 \phi}=0 \\
& \nabla^{2} \alpha+\left(1-\frac{1}{2} \alpha\right) t^{-2} \mathrm{e}^{2 \phi}-8 \alpha N \bar{N} \mathrm{e}^{-2 \phi}=0 .
\end{aligned}
$$

These equations are difficult to solve directly. However, we will show that in the firstorder formalism it naturally appears a quite simple structure, related to the $O(2,1) \sigma$-model, which automatically solves them.

Although not explicit, the appearance of singularities in the metric produces extra $\delta$ function sources, localized on the branch points, in the equations of motion (2.8). The $t$ dependence of the Riemann surface moduli is, therefore, provided by the covariant conservation of the 'underlying' energy-momentum tensor, which in turn implies the geodesic equations for the branch-point singularities.

\section{First-order formalism and the $O(2,1) \sigma$-model}

The first-order formalism gives a direct language for relating holonomies to the physical metric [16, 17]. For example, in [10] we have proposed a non-perturbative solution for the metric and the motion of $N$ interacting spinless particles in $(2+1)$ gravity, based on a harmonic mapping $X^{a}=X^{a}(t, z, \bar{z})$ from a regular coordinate system to Minkowskian multivalued coordinates.

Analogous to what we found for the gauge $K=0$, we are going to solve the $K=1 / 2 t$ gauge with a polydromic dreibein obeying the condition $\omega_{\mu, b}^{a}=0$ locally. This gauge choice 
allows us to define Minkowskian multivalued coordinates $X^{a} \equiv(T, Z, \bar{Z})$. Multivaluedness of $X^{a}$ is useful not only for introducing particles, but also for introducing topology. In fact the simplest definition of a genus, $g$, Riemann surface coupled to gravity is the quotient of a $(2+1)$ Minkowskian spacetime by a finite set of elements of the $I S O(2,1)$ Poincare group, i.e. we identify all the points that can be reached with isometries $\left(U_{i}, V_{i}, i=1, \ldots, g\right)$ of the Minkowskian metric $\mathrm{d} s^{2}=\mathrm{d} T^{2}-\mathrm{d} X^{2}-\mathrm{d} Y^{2}$ satisfying

$$
\prod_{i} U_{i} V_{i} U_{i}^{-1} V_{i}^{-1}=1
$$

This means that circling many times around the cycles $\left(a_{i}, b_{i}, i=1, \ldots, g\right)$ of the Riemann surface, the image of a point in the regular coordinate system by the $X^{a}$ mapping is a lattice of points in Minkowskian spacetime. The identification of this lattice with a point produces non-trivial topology.

To make contact with the physical metric, we need to build a representation of the $X^{a}$ 's starting from a regular coordinate system $x^{\mu}=(t, z, \bar{z})$, as follows:

$$
\mathrm{d} X^{a}=E_{\mu}^{a} \mathrm{~d} x^{\mu}=E_{0}^{a} \mathrm{~d} t+E_{z}^{a} \mathrm{~d} z+E_{\bar{z}}^{a} \mathrm{~d} \bar{z} .
$$

Here the dreibein $E_{\mu}^{a}$ is multivalued and satisfies the integrability condition :

$$
\partial_{[\mu} E_{v]}^{a}=0
$$

which implies a locally vanishing spin connection.

In order to have well defined $z$-coordinates, a Lorentz transformation should relate the values of $\mathrm{d} X^{a}$ 's circling around the cycles and circling around the branch points, so that the line element $\mathrm{d} s^{2}=\eta_{a b} \mathrm{~d} X^{a} \mathrm{~d} X^{b}$ is left single valued.

The conformal gauge choice can be implemented by parametrizing $E_{z}^{a}, E_{\bar{z}}^{a}$ in terms of null vectors:

$$
E_{z}^{a}=N W^{a}, \quad E_{\bar{z}}^{a}=\bar{N} \tilde{W}^{a},
$$

where $W^{2}=\tilde{W}^{2}=0$, and $N(z, t)$ should be a single-valued meromorphic function with poles at the branch points $z=\xi_{i}$. We have to build $W^{a}, \tilde{W}^{a}$ in order to represent the DJH [2] matching conditions (analogous to the particle case) of the $X^{a}$ coordinates, on the cycles $\left(C_{i}=a_{i}, C_{i+g}=b_{i}, i=1, \ldots, g\right)$ of the Riemann surface:

$$
\left(\mathrm{d} X^{a}\right)_{\mathrm{I}} \stackrel{C_{i}}{\longrightarrow}\left(\mathrm{d} X^{a}\right)_{\mathrm{II}}=\left(L_{i}\right)_{b}^{a}\left(\mathrm{~d} X^{b}\right)_{\mathrm{I}} \quad i=1,2, \ldots, 2 g
$$

where $L_{i}$ denotes the holonomies of the spin connection, i.e. the Lorentz part of the Poincaré holonomies $\left(U_{i}, V_{i}\right)$.

The simplest realization of such $O(2,1)$ monodromies is given by a spin $\frac{1}{2}$ projective representation:

$$
f(z, \bar{z}, t) \stackrel{C_{i}}{\longrightarrow} \frac{A_{i} f(z, \bar{z}, t)+B_{i}}{B_{i}^{*} f(z, \bar{z}, t)+A_{i}^{*}}, \quad\left|A_{i}\right|^{2}-\left|B_{i}\right|^{2}=1
$$

where the mapping function $f(z, \bar{z}, t)$ is an arbitrary function with branch cuts representing the Riemann surface and $A_{i}, B_{i}$ represent Minkowskian velocities, which are constants of motion.

The $W$ vectors, being in the adjoint representation of $O(2,1)$, can be built from the mapping function $f$, which is in the spin $\frac{1}{2}$ representation, as:

$$
W^{a}=\frac{1}{\partial_{z} f}\left(f, 1, f^{2}\right), \quad \tilde{W}^{a}=\frac{1}{\partial_{\bar{z}} \bar{f}}\left(\bar{f}, \bar{f}^{2}, 1\right) .
$$


The Minkowskian coordinates $X^{a}$ are obtained integrating the spatial components of the dreibein at constant time, along a generic path which does not intersect the branch cuts describing the Riemann surface on the $z$-plane:

$$
X^{a}=X_{1}^{a}+\int_{\left(\xi_{1}, \bar{\xi}_{1}\right)}^{(z, \bar{z})}\left(\mathrm{d} z E_{z}^{a}+\mathrm{d} \bar{z} E_{\bar{z}}^{a}\right) .
$$

Let us remark that we can completely solve the geodesic equations describing the branch-point trajectories, or equivalently the moduli trajectories in the first-order formalism, by measuring the distance between two branch points in the $X^{a}$ coordinates:

$$
X_{2}^{a}(t)-X_{1}^{a}(t)=\int_{\left(\xi_{1}, \bar{\xi}_{1}\right)}^{\left(\xi_{2}, \bar{\xi}_{2}\right)}\left(\mathrm{d} z N W^{a}(z, t)+\mathrm{d} \bar{z} \bar{N} \tilde{W}^{a}(\bar{z}, t)\right) .
$$

The explicit solution is obtained by inverting these relations to obtain the trajectories $\xi_{i}(t)$ as functions of the Poincaré holonomies, which are the constants of motion of the problem.

In order to determine the metric completely, we derive (3.8) with respect to time, and we obtain

$$
\begin{aligned}
E_{0}^{a} & =\partial_{t} X^{a} \\
& =\partial_{t} X_{1}^{a}+\partial_{t} \int_{\left(\xi_{1}, \bar{\xi}_{1}\right)}^{(z, \bar{z})}\left(\mathrm{d} z N W^{a}+\mathrm{d} \bar{z} \bar{N} \tilde{W}^{a}\right) \\
& =c^{a}(t)+\int_{\left(\xi_{1}, \bar{\xi}_{1}\right)}^{(z, \bar{z})}\left(\mathrm{d} z \partial_{t}\left(N W^{a}\right)+\mathrm{d} \bar{z} \partial_{t}\left(\bar{N} \tilde{W}^{a}\right)\right)
\end{aligned}
$$

In terms of the vectors $E_{0}^{a}=\partial_{t} X^{a}, E_{z}^{a}=N W^{a}, E_{\bar{z}}^{a}=\bar{N} \tilde{W}^{a}$, the components of the metric are given by :

$$
\begin{aligned}
& -2 g_{z \bar{z}}=\mathrm{e}^{2 \phi}=|N|^{2}(-2 W \tilde{W}), \\
& g_{0 z}=\frac{1}{2} \bar{\beta} \mathrm{e}^{2 \phi}=N W_{a} E_{0}^{a}, \quad g_{0 \bar{z}}=\frac{1}{2} \beta \mathrm{e}^{2 \phi}=\bar{N} \tilde{W}_{a} E_{0}^{a} \\
& g_{00}=\alpha^{2}-|\beta|^{2} \mathrm{e}^{2 \phi}=E_{0}^{a} E_{0}^{a}, \quad \alpha=n_{a} E_{0}^{a}
\end{aligned}
$$

To derive the expression for $\alpha$ we need to define the unit vector

$$
n^{a}=\frac{1}{1-|f|^{2}}\left(1+|f|^{2}, 2 \bar{f}, 2 f\right)=\epsilon_{b c}^{a} W^{b} \tilde{W}^{c}(W \tilde{W})^{-1},
$$

which represents the normal with respect to the surface $X^{a}=X^{a}(t, z, \bar{z})$, embedded at fixed time in the Minkowskian spacetime $\mathrm{d} s^{2}=\eta_{a b} \mathrm{~d} X^{a} \mathrm{~d} X^{b}$. The tangent plane is instead generated by the vectors

$$
\partial_{z} X^{a}=N W^{a}, \quad \partial_{\bar{z}} X^{a}=\bar{N} \tilde{W}^{a} .
$$

The $K=(2 t)^{-1}$ gauge condition can be rewritten in a first-order formalism as:

$$
K=(2 t)^{-1} \rightarrow E_{0}^{a}\left(\partial_{z} E_{\bar{z}}^{a}+\partial_{\bar{z}} E_{z}^{a}\right)=(2 t)^{-1} E_{0}^{a} n_{a} \mathrm{e}^{2 \phi} .
$$

Therefore, we must relax the condition that $E_{\mu}^{a}$ satisfies the Coulomb gauge as it happens in the $K=0$ gauge, and we require instead that

$$
\begin{aligned}
& \partial_{z} E_{\bar{z}}^{a}=\partial_{\bar{z}} E_{z}^{a}=\frac{1}{4 t} n^{a} \mathrm{e}^{2 \phi} \\
& n^{a}=\frac{1}{1-|f|^{2}}\left(1+|f|^{2}, 2 \bar{f}, 2 f\right) \quad\left(n^{a}\right)^{2}=1
\end{aligned}
$$


Can we solve this equation of motion? Yes, the answer can be written in several ways, i.e. by noticing that the vector $n^{a}$, satisfying the condition $\left(n^{a}\right)^{2}=1$, is a solution of the equation of motion of an $O(2,1) \sigma$-model, whose Lagrangian is:

$$
\mathcal{L}=\partial_{z} n^{a} \partial_{\bar{z}} n^{a} \quad\left(n^{a}\right)^{2}=1,
$$

or by noticing that the conformal factor can be represented in two ways resembling the solution of a Liouville-type equation:

$$
\mathrm{e}^{2 \phi}=\frac{N \bar{N}}{\partial_{z} f \partial_{\bar{z}} \bar{f}}\left(1-|f|^{2}\right)^{2}=16 t^{2} \frac{\partial_{z} \bar{f} \partial_{\bar{z}} f}{\left(1-|f|^{2}\right)^{2}} .
$$

It is not difficult to realize that this identity is generated by a basic first integral of the $O(2,1) \sigma$-model equations of motion:

$$
\frac{\partial_{z} f \partial_{z} \bar{f}}{\left(1-|f|^{2}\right)^{2}}=\frac{N(z)}{4 t}
$$

For future reference, we give the various forms on which the equations of motion of the Lagrangian (3.16) can be expressed:

$$
\partial_{z} \partial_{\bar{z}} n^{a}+\left(\partial_{z} n^{b} \partial_{\bar{z}} n_{b}\right) n^{a}=0 \quad \partial_{z} \partial_{\bar{z}} f+\frac{2 \bar{f} \partial_{z} f \partial_{\bar{z}} f}{\left(1-|f|^{2}\right)^{2}}=0 .
$$

The solution for $f$ in equation (3.18) has to respect monodromy conditions around each cycle $\left(a_{i}, b_{i}\right)$ in order that the Minkowskian coordinates $X^{a}$ represent the Poincaré group holonomies, which define the coupling of Riemann surfaces to gravity. In particular, the Lorentz group holonomies are in correspondence with $S U(1,1)$ Möbius transformations (3.6) that map the $f$-unit disk into itself.

The metric is instead a scalar with respect to the group $S U(1,1)$. Since every Riemann surface can be thought as an $n$-sheeted complex plane, the monodromy conditions around each branch point must be a cyclic subgroup of $S U(1,1)$. For example, since for a torus circling around each branch point twice we come back to the same point, the cyclic group is $Z_{2}$ which is represented by:

$$
f \rightarrow-f \text {. }
$$

The monodromy conditions for $f$ do not contain all the global information. To represent the translations, we need to play with the non-analyticity of the solution of the $\sigma$-model. As we shall see, if the holonomies are only Lorentz transformations, then $f$ becomes holomorphic. In the torus case, where we have to represent both Lorentz boosts and translations, a non-analytic dependence of the solution appears.

This remarkable connection between the $O(2,1) \sigma$-model and surfaces of constant mean curvature is analogous to what has been discussed in [18], for the Euclidean case.

In general every surface $\Sigma(t)$ (in this context a genus $g$ Riemann surface) immersed in a three-dimensional flat spacetime is characterized by its first and second fundamental form. By using conformal coordinates, the first form, i.e. the spatial metric, reads

$$
\mathrm{I}=\mathrm{e}^{2 \phi} \mathrm{d} z \mathrm{~d} \bar{z} .
$$

The second fundamental form is parametrized by $K$ and $N$ as follows:

$$
\begin{aligned}
& \mathrm{II}=K_{i j} \mathrm{~d} x^{i} \mathrm{~d} x^{j} \\
& K_{z z}=N \quad K_{z \bar{z}}=\frac{1}{2} K \mathrm{e}^{2 \phi} .
\end{aligned}
$$


Given such data of the surface, the position vector $X^{a}(z, \bar{z}, t)$, describing the foliation of Minkowski spacetime in terms of genus $g$ Riemann surfaces, can be found by solving the Gauss-Weingarten equations:

$$
\begin{aligned}
& \partial_{z}^{2} X^{a}=2 \partial_{z} \phi \partial_{z} X^{a}+N n^{a} \\
& \partial_{z} \partial_{\bar{z}} X^{a}=\frac{1}{2} K n^{a} \mathrm{e}^{2 \phi} \\
& \partial_{z} n^{a}=K \partial_{z} X^{a}+2 N \mathrm{e}^{-2 \phi} \partial_{\bar{z}} X^{a} .
\end{aligned}
$$

The integrability conditions of these equations, the Gauss and the Codazzi-Mainardi equations, take the form of the Einstein equations for $\phi$ and $N$. Then it is not difficult to show that, substituting the condition $K=(2 t)^{-1}$, these equations are solved by the $O(2,1)$ $\sigma$-model. Our mapping $f$ is called the Gauss map of the surface.

\section{Some solutions in conformal gauge}

In this section we shall apply the first-order formalism method to discuss some examples of the solution of the $O(2,1) \sigma$-model.

For the torus case, equation (3.1) becomes $U V=V U$ and it is solved by an Abelian subgroup $U(1) \otimes U(1)$ of the $I S O(2,1)$ Poincaré group, which can be taken as a Lorentz boost along the $x$ direction + a translation in the $y$ direction. The torus coupled to $(2+1)$ gravity is defined by the following holonomy transformations:

$U:\left\{\begin{array}{l}T \rightarrow \cosh \chi_{1} T+\sinh \chi_{1} X \\ X \rightarrow \cosh \chi_{1} X+\sinh \chi_{1} T \\ Y \rightarrow Y+\omega_{1}\end{array} \quad V:\left\{\begin{array}{l}T \rightarrow \cosh \chi_{2} T+\sinh \chi_{2} X \\ X \rightarrow \cosh \chi_{2} X+\sinh \chi_{2} T . \\ Y \rightarrow Y+\omega_{2}\end{array}\right.\right.$.

Starting from a generic point $\left(T_{0}, X_{0}, Y_{0}\right)$ and applying the transformations $(U, V)$ connected to the cycles $(a, b)$ of the torus, we obtain a lattice of points which belong to the surface

$$
T^{2}-X^{2}=T_{0}^{2}-X_{0}^{2}=C .
$$

At a given $C$ such a surface again describes a torus, and the spacetime evolution is simply obtained by allowing a time-dependent constant $C=C(t)$, which in the York time gauge is

$$
C=t^{2}=T^{2}-X^{2} .
$$

After a transformation of the Minkowskian coordinates

$$
T=t \cosh \frac{U}{t} \quad X=t \sinh \frac{U}{t},
$$

we obtain a plane lattice in the $(U, Y)$ coordinates, which is analogous to a static torus. The rescaling factor $1 / t$ in front of $U$ is necessary to keep the spatial metric in conformal gauge:

$$
\mathrm{d} s^{2}=\left(1-\frac{U^{2}}{t^{2}}\right) \mathrm{d} t^{2}+2 \frac{U}{t} \mathrm{~d} U \mathrm{~d} t-\mathrm{d} U^{2}-\mathrm{d} Y^{2},
$$

from which $\alpha=1, \beta=U / t$ is real and $\mathrm{e}^{2 \phi}=1$.

The variable $\tilde{Z}=U+\mathrm{i} Y$ has a simple translation monodromy:

$$
\tilde{Z} \stackrel{a}{\longrightarrow} \tilde{Z}+\chi_{1} t+\mathrm{i} \omega_{1} \quad \tilde{Z} \stackrel{b}{\longrightarrow} \tilde{Z}+\chi_{2} t+\mathrm{i} \omega_{2} .
$$



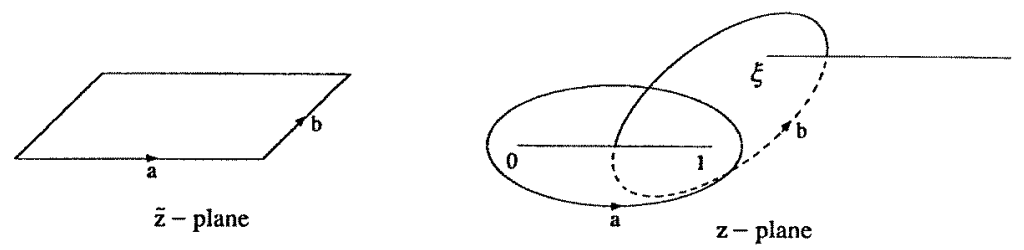

Figure 1. The Minkowskian and the branch cut representation of a torus.

It can be represented as a standard Abelian integral on a $z$-plane with two branch cuts (see figure 1)

$$
\tilde{Z}=\int_{0}^{z} \frac{\mathrm{d} z}{w(z, t)} \quad w^{2}(z, t)=R(t) z(z-1)(z-\xi(t)),
$$

where the position of the third singularity $\xi(t)$ is time-dependent, in order to represent the translation monodromies (4.6).

Therefore, the solution for the torus is given by the following mapping:

$$
\begin{aligned}
& T=t \cosh \left[\frac{1}{2 t}\left(\int_{0}^{z} \frac{\mathrm{d} z}{w(z, t)}+\int_{0}^{\bar{z}} \frac{\mathrm{d} \bar{z}}{\bar{w}(\bar{z}, t)}\right)\right]=t \cosh \phi(z, \bar{z}, t) \\
& X=t \sinh \left[\frac{1}{2 t}\left(\int_{0}^{z} \frac{\mathrm{d} z}{w(z, t)}+\int_{0}^{\bar{z}} \frac{\mathrm{d} \bar{z}}{\bar{w}(\bar{z}, t)}\right)\right]=t \sinh \phi(z, \bar{z}, t) \\
& Y=\frac{1}{2 \mathrm{i}}\left(\int_{0}^{z} \frac{\mathrm{d} z}{w(z, t)}-\int_{0}^{\bar{z}} \frac{\mathrm{d} \bar{z}}{\bar{w}(\bar{z}, t)}\right) .
\end{aligned}
$$

The Poincare holonomies (4.1) tell us that the motion of the branch points is trivial in the $X^{a}$ coordinates:

$$
\begin{aligned}
& X^{a}(0)=(t, 0,0) \\
& X^{a}(1)=\left(\gamma(1) t, \gamma(1) \beta(1) t, b_{1}\right) \\
& X^{a}(\xi)=\left(\gamma(2) t, \gamma(2) \beta(2) t, b_{2}\right) .
\end{aligned}
$$

Taking for example the particle in 0 at rest, the particle in 1 is moving in the $x$-direction with constant velocity $\beta(1)=\tanh \frac{1}{2} \chi_{1}$ and impact parameter $b_{1}=\frac{1}{2} \omega_{1}$. Analogously the particle in $\xi$ is moving in the $x$-direction with velocity $\beta(2)=\tanh \frac{1}{2} \chi_{2}$ and impact parameter $b_{2}=\frac{1}{2} \omega_{2}$. In the $z$-coordinates, only the motion of the third singularity, $\xi(t)$, is necessary up to global rescalings of the $z$-coordinate, while the other two can remain at rest.

It is straightforward to derive the equations of motion for the modulus of the torus and for the area, which take the usual form [7,8]:

$$
\tau(t)=\frac{\chi_{1} t+\mathrm{i} \omega_{1}}{\chi_{2} t+\mathrm{i} \omega_{2}} \quad A(t)=\int \mathrm{d} z \mathrm{~d} \bar{z} \mathrm{e}^{2 \phi}=t\left(\chi_{1} \omega_{2}-\chi_{2} \omega_{1}\right) .
$$

The motion of the modulus is essentially a consequence of the free motion in Minkowskian coordinates of the branch points. It has also to satisfy another consistency condition [8], namely that the motion of the moduli must be geodesic with respect to the natural metric of the moduli space, the Weil-Petersson metric, which in the case of the torus is equivalent to the Poincaré metric of the upper half plane:

$$
\mathrm{d} s^{2}=\frac{\mathrm{d} \tau \mathrm{d} \bar{\tau}}{(\operatorname{Im} \tau)^{2}} \text {. }
$$


The form (4.10) of the solution for the modulus is consistent since it describes a circular arc in the moduli space, which is a geodesic of the Poincaré metric.

The corresponding dreibein, $E_{\mu}^{a}=\partial_{\mu} X^{a}$, is given by $(a=(t, x, y))$ :

$$
\begin{aligned}
& E_{z}^{a}=\frac{1}{2 t w(z, t)}(X, T,-\mathrm{i} t) \\
& E_{0}^{a}=\left(\frac{T}{t}+X \frac{\partial \phi}{\partial t}, \frac{X}{t}+T \frac{\partial \phi}{\partial t}, \frac{\partial Y}{\partial t}\right) .
\end{aligned}
$$

The conformal gauge condition $\left(E_{z}^{a}\right)^{2}=0$ is verified due to equation (4.3).

From equation (4.12) we can read the solution for $N$ and $f$ satisfying equation (3.18):

$$
\begin{aligned}
& f(z, \bar{z}, t)=\frac{X}{t+T}=\tanh \frac{\phi(z, \bar{z}, t)}{2} \\
& N(z, t)=\frac{1}{4 t w^{2}(z, t)} .
\end{aligned}
$$

So we have found that, for the special case of boosts along the $x$-axis, $f$ is real and $N$ is related to the quadratic holomorphic differential. In general, we can guess that $N(z, t)$ is a known source for equation (3.18), being a combination of the quadratic holomorphic differentials of the Riemann surface.

The function tanh can also be expected since it diagonalizes the monodromy conditions for $f$ around the cycles $C_{i}=(a, b)$ of the torus:

$f \stackrel{C_{i}}{\longrightarrow} \frac{A_{i} f+B_{i}}{B_{i} f+A_{i}} \quad A_{i}=\cosh \frac{\chi_{i}}{2} \quad B_{i}=\sinh \frac{\chi_{i}}{2} \quad i=1,2$.

The linear dependence of its argument from the Abelian integrals represents the change of sign $f \rightarrow-f$ around each branch point.

Let us integrate equation (2.8), which specifies the spatial data, on the two-dimensional surface:

$\int \nabla^{2} \phi \mathrm{d} z \mathrm{~d} \bar{z}=\oint \vec{\nabla} \phi \cdot \vec{n} \mathrm{~d} l=4 \int \frac{\partial_{z} f \partial_{\bar{z}} \bar{f}-\partial_{z} \bar{f} \partial_{\bar{z}} f}{\left(1-|f|^{2}\right)^{2}} \mathrm{~d} z \mathrm{~d} \bar{z}=4 \int \frac{\mathrm{d} f \mathrm{~d} \bar{f}}{\left(1-|f|^{2}\right)^{2}}$.

The line integral has to be done around all the singularities on the $z$-plane. In the case of the torus the power behaviour of $\mathrm{e}^{2 \phi}$ around each singularity ensures that the contour integral is finite. Then the last relation says that the modulus $|f|^{2}$ is always limited inside the unit disk $\left(|f|^{2}<1\right)$. This is an important property of this temporal splicing, since then we are safe from spurious singularities and the determinant of the metric is always non-vanishing.

Such integral property must be essential to prove that in this gauge the foliation for every Riemann surface is well defined, once suitable boundary conditions for the singular behaviour of $\mathrm{e}^{2 \phi}$ are imposed, such as a particle-like singularity with a power behaviour.

Since $f$ is real in the case of the torus and its image must be contained inside the unit disk, we conclude that $f$ maps the real variable $\phi(z, \bar{z}, t)$ inside the real diameter $D=[-1,1]$. Since $f$ is polydrome, it can be restricted to cover a segment of $D$. Circling many times around each cycle of the torus, $\operatorname{Im} f$ gives a tessellation of the diameter.

This particular feature of the torus should be valid in general. For every Riemann surface the image of the Gauss map can be restricted to a polygon inside the unit disk. Circling many times around each cycle we should obtain a tessellation of the unit disk, instead of the diameter $D$, as a consequence of the non-Abelian relation (3.1) which replaces the Abelian one $U V=V U$ for the torus. An example of tessellation of the unit disk with curvilinear triangles is given in figure 2 [19]. 


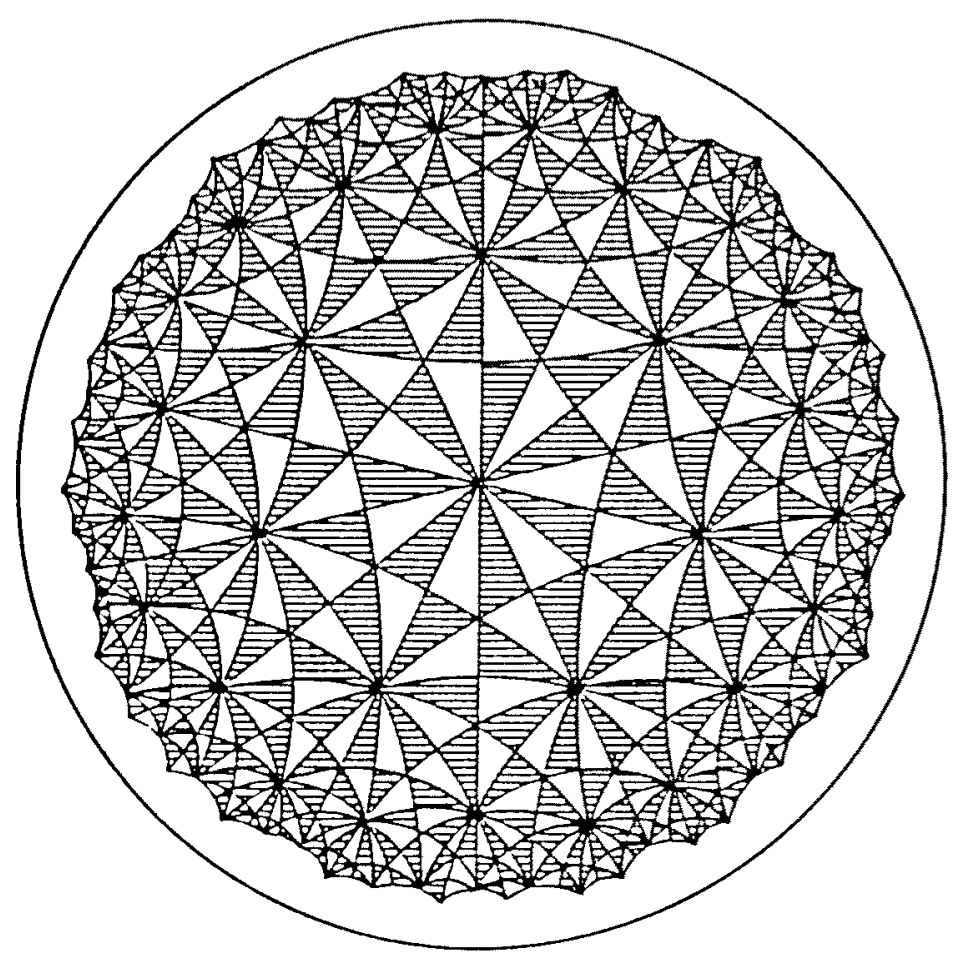

Figure 2. Tesselation of the unit disk with hypergeometric functions.

There is another limit in which equation (3.18) can be solved, i.e. when we consider the Gauss map, $f$, depending on a single variable $f=f(\bar{z})$. We shall show that, in this case, the basic relation (3.1) defining the Riemann surface evolution is solved by representing the $U_{i}$ and $V_{i}$ holonomies with arbitrary boost but no translations. This is a totally different case from Moncrief's solution for the torus, which contains mixed Lorentz boost and translation holonomies.

This case corresponds to $N=0[1,8]$, and the equation (3.18) can have a meaning, as $N \rightarrow 0, \partial_{z} f \rightarrow 0$ but the ratio

$$
\frac{N}{\partial_{z} f}=4 t \frac{\partial_{z} \bar{f}}{\left(1-|f|^{2}\right)^{2}}
$$

still finite. From this identity, the dreibein reads:

$$
E_{z}^{a}=4 t \frac{\partial_{z} \bar{f}}{\left(1-|f|^{2}\right)^{2}}\left(f, 1, f^{2}\right) .
$$

It gives rise to a Minkowskian transformation of the type:

$$
T=2 t \frac{1+|f|^{2}}{1-|f|^{2}}, \quad Z=4 t \frac{\bar{f}}{1-|f|^{2}} .
$$

The particular relation (4.18) between Minkowskian coordinates and $f$ realizes explicitly the isomorphism between $S O(2,1)$ and $S U(1,1)$, and the Möbius transformations of $f$ correspond to pure Lorentz transformation holonomies for the $X^{a}$ coordinates. 
We easily recognize that the Minkowskian coordinates satisfy the constraint:

$$
T^{2}-Z \bar{Z}=4 t^{2} \text {. }
$$

The same surface can be obtained starting from a generic point $\left(T_{0}, Z_{0}, \bar{Z}_{0}\right)$ and applying all the $S O(2,1)$ movements related to the cycles $\left(a_{i}, b_{i}\right)$. Hence this time foliation is natural since it is induced by the Poincaré holonomies.

The $X=X(x)$ mapping (4.18) produces the hyperbolic metric on the disk:

$$
\mathrm{d} s^{2}=4 \mathrm{~d} t^{2}-16 t^{2} \frac{\mathrm{d} f \mathrm{~d} \bar{f}}{\left(1-|f|^{2}\right)^{2}},
$$

from which we can read $\alpha=2, \beta=0$ and $\mathrm{e}^{2 \phi}=16 t^{2} /\left(1-|f|^{2}\right)^{2}$ which solve equation (2.8).

The conformal mapping $f(\bar{z})$ still has to be determined. First, we remember that every genus $g$ Riemann surface is determined by a fundamental group $\pi_{1}(\Sigma)$ generated by the $2 g$ holonomies $\left(U_{i}, V_{i}\right)$ satisfying the relation (3.1). Let us denote with $H$ the unit disk with its metric of negative constant curvature. The group $S U(1,1)$ acts on it, maintaining its metric. Consider a subgroup of it $\Gamma \subset S U(1,1)$, isomorphic to $\pi_{1}(\Sigma)$, the quotient $H / \Gamma$ is a Riemann surface of genus $g$, with the same constant curvature metric of the unit disk $H$.

Therefore, we can think that the image of the conformal mapping, $f$, gives a tessellation of the unit disk on which the holonomy acts as $S U(1,1)$ and we can restrict the fundamental region of $\operatorname{Im} f$ to be inside a closed geodesic $4 g$-gon of the unit disk with hyperbolic metric, where the conformal mapping becomes one to one.

For pure $S O(2,1)$ holonomies, the equations of motion for the branch points are simply:

$$
X^{a}\left(\xi_{i}\right)-X^{a}\left(\xi_{j}\right)=\left(V_{i}-V_{j}\right) t .
$$

Since the $X^{a}=X^{a}(x)$ mapping in equation (4.18) is also linear in $t$, we conclude that there is no evolution in the $z$-coordinate for the branch points. As a consequence, there is no time evolution for the moduli [8], and the only time dependence comes from the overall scale factor, $t^{2}$, in equation (4.20). We can suppose that the branch points, $\xi_{i}$, have fixed positions on the real axis. Then to find the Gauss map $f$ the theorem of complex analysis is helpful: given a simple and closed geodesic polygon $\Pi$ of the Poincare metric in the upper half $w$-plane, whose sides are circular arcs making angles $\alpha_{1} \pi, \alpha_{2} \pi, \ldots, \alpha_{n} \pi$, at the vertices $A_{1}, A_{2}, \ldots, A_{n}$ where $0 \leqslant \alpha_{j} \leqslant 2$, then there exist real numbers $\xi_{1}, \xi_{2}, \ldots, \xi_{n}$, $\beta_{1}, \beta_{2}, \ldots \beta_{n}$ such that

$$
\begin{aligned}
& \xi_{1}<\xi_{2}<\cdots<\xi_{n}, \quad \sum_{j=1}^{n} \beta_{j}=0 \\
& \sum_{j=1}^{n}\left(2 \beta_{j} \xi_{j}+1-\alpha_{j}^{2}\right)=0, \quad \sum_{j=1}^{n}\left(\beta_{j} \xi_{j}^{2}+\left(1-\alpha_{j}^{2}\right) \xi_{j}\right)=0
\end{aligned}
$$

and the upper $z$-plane is conformally mapped inside $\Pi$ by

$$
w(z)=\frac{u_{1}(z)}{u_{2}(z)}
$$

where $u_{1}(z)$ and $u_{2}(z)$ are two linearly independent solutions of the Fuchsian differential equation:

$$
u^{\prime \prime}+\left[\frac{1}{4} \sum_{j=1}^{n} \frac{1-\alpha_{j}^{2}}{\left(z-\xi_{j}\right)^{2}}+\frac{1}{2} \sum_{j=1}^{n} \frac{\beta_{j}}{z-\xi_{j}}\right] u=0 .
$$


The points $\xi_{j}$ correspond to the vertices $A_{j}$. A simple conformal mapping relates the upper $w$-plane endowed with the Poincaré metric to the $f$-unit disk with the hyperbolic metric.

Let us remark that in order to obtain the tessellation property of the unit disk, the angles $\alpha_{i} \pi$ must be chosen as $\pi\left(1-1 / n_{i}\right)$, with $n_{i}$ positive integers. Such a mapping problem has been investigated in connection with Fuchsian groups and functions. In fact, to each geodesic polygon of the $f$-unit disk, whose angles in the vertices have the measure $\pi\left(1-1 / n_{i}\right)$, with $n_{i}$ positive integers, a discrete group of movements $\Gamma$ and an analytic function $z(f)$ defined in the $f$-unit disk is connected, which is invariant under the $\Gamma$ action on the $f$ variable. The $\Gamma$ group of $S U(1,1)$ Möbius transformations on the disk is called the Fuchsian group. The function $z(f)$ is a Fuchsian function with respect to the group $\Gamma$. Instead the inverse $f=f(z)$ is polydrome, and it can be restricted to mapping the $z$-plane into the geodesic polygon. Therefore, we conclude that our conformal mapping is the inverse of such a Fuchsian function $z(f)$. Examples of them can be built from the series of the form (Poincaré series):

$$
\Theta(f)=\sum_{i=1}^{\infty} \frac{H(f)}{\left(\bar{b}_{i} f+\bar{a}_{i}\right)^{2 m}},
$$

where $m$ is a positive integer, $H(f)$ is a rational function in the geodesic polygon and the sum is extended to all the elements of the Fuchsian group, generated by the holonomies. This series has the following Poincaré property:

$$
\Theta\left(\frac{a_{k} f+b_{k}}{\bar{b}_{k} f+\bar{a}_{k}}\right)=\left(\bar{b}_{k} f+\bar{a}_{k}\right)^{2 m} \Theta(f) \quad \forall\left(a_{k}, b_{k}\right) \in \Gamma .
$$

This means that it has a very simple transformation rule under the action of the Fuchsian group. Taking the ratio of two Poincaré series with the same index $m$

$$
z(f)=\frac{\Theta_{1}(f)}{\Theta_{2}(f)}
$$

we obtain a Fuchsian function, which is invariant under the action of the Fuchsian group

$$
z\left(\frac{a_{k} f+b_{k}}{\bar{b}_{k} f+\bar{a}_{k}}\right)=z(f) \Longleftrightarrow z\left(X^{a}\right)=z\left(\Lambda_{b}^{(k) a} X^{b}\right) \quad \forall k .
$$

Maybe the special functions which integrate the whole $O(2,1) \sigma$-model have to be searched as non-analytic generalizations of such a series, with the property

$$
z\left(X^{a}\right)=z\left(\Lambda_{b}^{(k) a} X^{b}+q^{(k) a}\right) \quad \forall k
$$

in which one has to sum over all the group elements generated by the Poincaré holonomies $\left(\Lambda_{b}^{(k) a}, q^{(k) a}\right)$.

However, only an analytic function is inequivocally defined by its monodromy properties. For the general case we expect that finding new solutions is a difficult question.

\section{Discussion}

We have shown here that the first-order formalism, that has allowed us to solve the $\mathrm{N}$-body problem, can be extended to the case of Riemann surfaces in $(2+1)$ gravity. We have found that the $O(2,1) \sigma$-model solves explicitly all the Einstein equations. The solution is characterized by an analytic function $N(z)$ which is the component $K_{z z}$ of the extrinsic curvature tensor and by the Gauss map $f(z, \bar{z}, t)$. This function $f$ maps a complex plane with branch cuts into the unit disk with hyperbolic metric. The holonomies of $f$ are elements 
of $S U(1,1)$ and isometries of this metric. Therefore, we can delimit $\operatorname{Im} f$ into a geodesic polygon inside the unit disk. This property is also true for the conformal mapping $f$ of our $N$-particle solution [10]. The peculiar signal of topology should be that this mapping produces a tessellation of the unit disk, a property which is not generally true for the particle case.

We have discussed in [20] that the line defined by $|f|=1$ is a spurious singularity of the conformal gauge, since the metric determinant vanishes there, and also the boundary for the existence of closed time-like curves (CTC) [21]. Therefore, our qualitative analysis on the behaviour of the mapping function $f$ suggests that the Riemann surfaces solutions should not suffer from the CTC problem.

We have given explicit solutions for the mapping function $f$ for the torus and for all Riemann surfaces having $S O(2,1)$ holonomies. It turns out that in both cases the inverse mapping $z=z(f)$ is a single-valued function, i.e. an automorphic function. The $N(z)$ function for the torus is related to the quadratic holomorphic differential, a property that is probably true for all Riemann surfaces.

We have found that the Poincaré holonomies have a quite simple particle interpretation. They determine the evolution for the branch points, which move freely in the Minkowskian $X$ coordinates according to

$$
X^{a}\left(\xi_{i}\right)-X^{a}\left(\xi_{j}\right)=\left(V_{i}^{a}-V_{j}^{a}\right) t+B_{i}^{a}-B_{j}^{a} .
$$

The moduli trajectories, which have to be geodesic of the metric of Teichmuller space, should be a consequence of the trajectories of the branch points.

The next step would be to find a quantization scheme which takes into account this classical reduction of three-dimensional gravity in two-dimensional field theories.

\section{Acknowledgments}

I would like to thank M Ciafaloni and $\mathrm{R}$ Loll for valuable discussions. I am particularly grateful to Professor M Ciafaloni for having introduced me to the problem of $(2+1)$ gravity on the torus, where he first found an independent solution in the gauge $K=0$ [13].

\section{Appendix. Einstein equations from the $O(2,1) \sigma$-model}

Let us verify that the second-order equations of motion are reproduced by the first-order equations of motion.

For example, to obtain equation (2.8) we have to show that

$$
\partial_{z} \partial_{\bar{z}} \phi+\frac{\partial_{z} f \partial_{\bar{z}} f-\partial_{z} \bar{f} \partial_{\bar{z}} f}{\left(1-|f|^{2}\right)^{2}}=0 .
$$

From the definition of $\phi$ :

$$
\phi=\frac{1}{2} \log \partial_{z} \bar{f} \partial_{\bar{z}} f-\log \left(1-|f|^{2}\right)
$$

making use of the $\sigma$-model equation of motion for $f$ (equation (3.19)), we can easily obtain (A.1).

Let us compute the equation of motion for $\alpha$. By definition

$$
\partial_{z} \partial_{\bar{z}} \alpha=\partial_{z} \partial_{\bar{z}}\left(n^{a} E_{a}^{0}\right)=\left(\partial_{z} \partial_{\bar{z}} n^{a}\right) E_{a}^{0}+\partial_{z} n^{a} \partial_{\bar{z}} E_{a}^{0}+\partial_{\bar{z}} n^{a} \partial_{z} E_{a}^{0}+n^{a} \partial_{z} \partial_{\bar{z}} E_{a}^{0} .
$$


We can easily compute the first term which is exactly the $\sigma$-model equation for $n^{a}$ (equation (3.19)):

$\partial_{z} \partial_{\bar{z}} n^{a}=-\left(\partial_{z} n^{b} \partial_{\bar{z}} n_{b}\right) n^{a}=2 \frac{\partial_{z} \partial_{\bar{z}} \bar{f}+\partial_{z} \bar{f} \partial_{\bar{z}} f}{\left(1-|f|^{2}\right)^{2}} n^{a}=\left(2 N \bar{N} \mathrm{e}^{-2 \phi}+\frac{1}{2 t^{2}} \mathrm{e}^{2 \phi}\right) n^{a}$,

from which

$\nabla^{2} \alpha=\left(8 N \bar{N} \mathrm{e}^{-2 \phi}+\frac{1}{2 t^{2}} \mathrm{e}^{2 \phi}\right) \alpha+4 n^{a} \partial_{z} \partial_{\bar{z}} E_{a}^{0}+4 \partial_{\bar{z}} n^{a} \partial_{z} E_{a}^{0}+4 n^{a} \partial_{z} \partial_{\bar{z}} E_{a}^{0}$.

Let us compute the residual contribution:

$\partial_{z} E_{a}^{0}=\partial_{z} \partial_{0} X_{a}=\partial_{0} E_{z}^{a}=\left[\partial_{0} \log N-\partial_{0} \log \partial_{z} f-\frac{2 \partial_{0} f \bar{f}}{\left(1-|f|^{2}\right)}\right] X^{a}+N \frac{\partial_{0} f}{\partial_{z} f} n^{a}$.

Similarly, the spatial derivative of $n^{a}$ can be decomposed as a sum of the two orthogonal vectors $W^{a}$ and $\tilde{W}^{a}$ :

$\partial_{z} n^{a}=\frac{2 \partial_{z} f \partial_{\bar{z}} \bar{f}}{\left(1-|f|^{2}\right)^{2}} \tilde{W}^{a}+\frac{2 \partial_{z} f \partial_{z} \bar{f}}{\left(1-|f|^{2}\right)^{2}} W^{a}=\frac{1}{2 t} X^{a}+\frac{2 \partial_{z} f \partial_{\bar{z}} \bar{f}}{\left(1-|f|^{2}\right)^{2}} \tilde{W}^{a}$,

from which

$\partial_{z} n^{a} \partial_{\bar{z}} E_{a}^{0}+\partial_{\bar{z}} n^{a} \partial_{z} E_{a}^{0}=\frac{1}{2 t} N \bar{N} W \tilde{W} \partial_{0}\left[\log \frac{N \bar{N}}{\partial_{z} f \partial_{\bar{z}} \bar{f}}\left(1-|f|^{2}\right)^{2}\right]=-\frac{1}{4 t} \partial_{t} \mathrm{e}^{2 \phi}$.

The last contribution is given by:

$n^{a} \partial_{z} \partial_{\bar{z}} E_{a}^{0}=n^{a} \partial_{t}\left(\partial_{z} \partial_{\bar{z}} X^{a}\right)=n^{a} \partial_{t}\left(\frac{1}{4 t} n^{a} \mathrm{e}^{2 \phi}\right)=-\frac{1}{4 t^{2}} \mathrm{e}^{2 \phi}+\frac{1}{4 t} \partial_{t} \mathrm{e}^{2 \phi}$.

Summing the various contributions, we reproduce equation (2.8).

\section{References}

[1] Witten E 1988 Nucl. Phys. B 31146 Witten E 1989 Nucl. Phys. B 323113

[2] Deser S, Jackiw R and 't Hooft G 1984 Ann. Phys., NY 152220

[3] Gott J R and Alpert M Gen. Rel. Grav. 16243 Gott J R 1985 Astrophys. J. 288422

[4] 't Hooft G 1988 Commun. Math. Phys. 117685

[5] Nelson J E and Regge T 1991 Commun. Math. Phys. 141211 Nelson J E and Regge T 1992 Int. J. Mod. Phys. B 62091 Nelson J E and Regge T 1992 Class. Quantum Grav. 9 S187

[6] Moncrief V 1989 J. Math. Phys. 302907 Moncrief V 1990 J. Math. Phys. 312978

[7] Carlip S 1989 Nucl. Phys. B 324106

Carlip S 1990 Phys. Rev. D 422647

Carlip S 1992 Phys. Rev. D 464387

Carlip S 1993 Class. Quantum Grav. 10207

[8] Hosoya A and Nakao K 1990 Prog. Theor. Phys. 84739 Hosoya A and Nakao K1990 Class. Quantum Grav. 7163

[9] Bellini A and Valtancoli P 1955 Phys. Lett. 348B 44 Bellini A, Ciafaloni M and Valtancoli P 1995 Nucl. Phys. B 454449

[10] Bellini A, Ciafaloni M and Valtancoli P 1995 Phys. Lett. B 357532 Bellini A, Ciafaloni M and Valtancoli P 1996 Nucl. Phys. B 462453

[11] Welling M 1996 Class. Quantum Grav. 13653

[12] Fuchs R 1907 Mater. Ann. 63301 
[13] Ciafaloni M 1996 Preprint hep-th/9612219

[14] Arnowitt R, Deser S and Misner C W 1962 Gravitation: An Introduction to Current Research ed L Witten (New York: Wiley)

[15] Hanson A J, Regge T and Teitelboim C 1976 Constrained Hamiltonian Systems (Rome: Accademia Nazionale dei Lincei)

[16] Cappelli A, Ciafaloni M and Valtancoli P 1992 Nucl. Phys. B 369669

Cappelli A, Ciafaloni M and Valtancoli P 1991 Phys. Lett. B 273431

[17] Grignani G and Nardelli G 1991 Phys. Lett. B 26445

Grignani G and Nardelli G 1992 Nucl. Phys. B 370491

[18] Ody M S and Ryder L H 1995 Int. J. Mod. Phys. 10337

Puzio R S 1994 Class. Quantum Grav. 112667

[19] Yoshida M 1987 Fuchsian Differential Equations Max-Planck-Institut für Mathematik (Bonn: Fried)

[20] Ciafaloni M and Valtancoli P 1996 Preprint hep-th/9609105

[21] Gott J R 1991 Phys. Rev. Lett. 661126

Deser S, Jackiw R and 't Hooft G 1992 Phys. Rev. Lett. 682647

Menotti P and Seminara D 1992 Phys. Lett. B 30125 